\title{
Optical Strategies for Generating and Transmitting Signals in OFDM-RoF Systems
}

\author{
Vitor Lopes $^{2}$, Diogo Bento ${ }^{2}$, Maria C.R. Medeiros ${ }^{1}$, and Paula Laurêncio ${ }^{1,2}$ \\ ${ }^{1}$ IT-Institute of Telecommunications, Av. Rovisco Pais 1, 1049-001, Lisboa, Portugal \\ ${ }^{2}$ Instituto Superior de Engenharia, Universidade do Algarve, 8005 Faro, Portugal \\ vlopes@ualg.pt, db_eee@yahoo.com, \{cmedeiro,plaurenc\}@ualg.pt
}

\begin{abstract}
Radio-over-Fiber (RoF) systems which act as backbone networks are very promising means of reducing overall costs of $60 \mathrm{GHz}$ WPANs, as well leading to networks with limited intercellular interference. The main objective of this paper is to compare the performance of two systems that generate Optical Single Side Band (OSSB) OFDM signals at the mm-wave band with and without optical up-conversion. Both systems use a DD-MZM: one is a standard model, whereas the other is a DD-MZM cascaded with a phase modulator (PM). The comparative study presented here also includes practical impairments such as the finite extinction ratio of DD-MZMs.
\end{abstract}

Keywords: Radio over fiber, Orthogonal Frequency Division Multiplexing, 60 $\mathrm{GHz}$, fiber chromatic dispersion, Optical Single Side Band,optical up-conversion.

\section{Introduction}

The $60 \mathrm{GHz}$ (mm-wave) band has been allocated worldwide for short range wireless communications due to its inherent high propagation losses. The abundant unlicensed spectrum around the $60 \mathrm{GHz}$ has the potential to support consumer demands for indoor wireless applications that require high bandwidth such as real-time streaming content download for high-definition TV, wireless gigabit Ethernet, etc. Due to the small coverage area, $60 \mathrm{GHz}$ networks require a large number of base stations (BSs) to cover a service area. This requirement has led to the development of system architectures where functions such as routing, signal processing, handover and frequency allocation are performed at the central office (CO). The best solution for connecting the $\mathrm{CO}$ with BSs in such radio network is via an optical fiber network, now known as radio over fiber $(\mathrm{RoF})$ [1].

Due to the high data rates of broadband wireless systems, we can have severe time-dispersion effects associated to the multipath propagation. Multicarrier (MC) modulation schemes combined with frequency-domain receiver implementations, especially the ones belonging to the orthogonal frequency division multiplexing (OFDM) class are widely used in several broadband wireless communication systems which have to deal with strong frequency-selective fading channels; as such, they are 
considered a good contender for this application. However, the optical distribution of high bit rate mm-wave signals is susceptible to fiber chromatic dispersion, which severely limits the transmission distance. This shortcoming can be mitigated using optical single-sideband (OSSB) modulation [2]. Several techniques have been proposed to generate mm-wave optical signals. Among them, optical frequency upconversion has the potential to provide a simple and cost efficient solution [3]. The principle of optical frequency up-conversion is to generate higher-order optical harmonics using an external nonlinear modulator, typically a dual-drive Mach-Zehnder modulator (DD-MZM) driven by a low frequency electrical signal. The efficiency of this technique relies not only on its capacity to maximize the required high-order harmonic, but also on its robustness against fiber dispersion. Therefore, the objective of this paper is to compare two systems that generate OSSB signals at the mm-wave band with and without optical up-conversion. Both systems use a DD-MZM: one is a standard model and other is a DD-MZM cascaded with a phase modulator (PM) [4].

The remainder of this paper is organized as follows. In section 2 we discuss the relationship to the Internet of things. In section 3, we present the network architecture. In section 4 , we present the simulation results and finally, in section 5, we conclude the paper.

\section{Relationship to Internet of Things}

Nowadays, high bandwidth services are supported by wired networks based on fiber optic technologies. However, consumer habits are increasingly changing; as such, users want to be able to use their mobile terminals and enjoy the same user experience as they do when connected to their fixed networks. The abundant unlicensed frequency spectrum around $60 \mathrm{GHz}$ offers the potential for multi-gigabit indoor Wireless Personal Area Networks, removing the bandwidth limitation of the present wireless networks.

The current generation of Wi-Fi systems (IEEE 802.11n) can achieve theoretically $600 \mathrm{Mb} / \mathrm{s}$, but new radio standards allowing higher data rates have emerged or are under development. All these new standards address the unlicensed mm-wave band from 57 to $66 \mathrm{GHz}$, in this band, divided into four $2.16 \mathrm{GHz}$-bandwidth channels, it is possible to achieve bit rates up to $7 \mathrm{~Gb} / \mathrm{s}$ per channel [5]. IEEE 802.15.3c[6] and WirelessHD standards were finalized in 2009 and are devoted to wireless personal area network communications.More recently, the IEEE 802.11ad group has been created by WiGig consortium: it addresses networking with wireless local area network systems and targets Wi-Fi Alliance certification. $60 \mathrm{GHz}$ technologies are now mature: WirelessHD devices are already available in the market for wireless highdefinition multimedia interface (HDMI) applications and IEEE 802.11ad products.

Due to the high-propagation attenuation and the fact that signals in the $60 \mathrm{GHz}$ band cannot cross walls,the premises should be connected employing optical fiber technology. Consequently, $60 \mathrm{GHz}$ networks require an extensive high capacity feeder network; thisdemands an optical fiber network thatprovides the required performance, while being cost-effective. 
Currently, wired and wireless services are separately provided by two independent physical networks. However, with the fast evolution of wireless technology, ubiquitous and always on wireless systems in buildings are expected to emerge in the near future. Hybrid broadband access networks combining wireless and wireline Wavelength Division Multiplexed Passive Optical Networks (WDM-PONs) networks can support the requirement of these future broadband ubiquitous networks. The target of a converged hybrid architecture is to connect, via a common optical WDM-PON infrastructure, both wireline and wireless users. Hybrid wireless WDM-PON architecture integrating mm-wave wireless services within WDM-PONs will be the future access networks.

\section{System Description}

The architecture of the $60 \mathrm{GHz}$ OFDM-RoF numerical simulation platform using the VPI Transmission Maker Simulator ${ }^{\circledR}$ is shown in figure 1 and 2. The difference between the two figures is the optical external modulator; the first one is a DD-MZM biased in quadrature, whilst the second one is a DD-MZM biased in quadrature cascaded with a phase modulator (PM), both of them generating an OSSB signal. The objective of the second configuration is to demonstrate that the effect of the finite extinction ratio of the DD-MZMs on OSSB modulation can be greatly reduced. The light source considered in the simulation is a laser with $1 \mathrm{MHz}$ of line width and $5 \mathrm{~mW}$ of emission power with $-150 \mathrm{~dB} / \mathrm{Hz}$ relative intensity noise (RIN). A $2.5 \mathrm{Gbit} / \mathrm{s}$ NRZ data is divided in 512 blocks. Each blockis presented as 512parallel data paths to the OFDM transmitter. These 512paths aremodulated onto 512equally-spaced subcarriers using 4 Quadrature-Amplitude Modulation(4QAM). The cyclic prefix (CP) is $12.5 \%$. Each QAM data channel is presented to an input of an IFFT that produces a complex-valued time domainwaveform containing a superposition of all of the subcarriers. This waveform therefore is modulated onto an electrical carrier, $\mathrm{f}_{\mathrm{c}}=60 \mathrm{GHz}$, using an I-Qmodulator, producing a real-valued signal. This carrier gives a band of optical OFDM sub-carriers with a bandwidth of $750 \mathrm{MHz}$ centered in the optical carrier at $193.1 \mathrm{THz}$ plus $60 \mathrm{GHz}$ after external modulation by a DD-MZM. Both DDMZMs were assumed to have a $6 \mathrm{~dB}$ insertion loss and a $30 \mathrm{~dB}$ extinction ratio $\left(\mathrm{R}_{\text {ext }}\right)$. The fiber attenuation was set to $0.2 \mathrm{~dB} / \mathrm{km}$ and the dispersion coefficient to $16 \mathrm{ps} /(\mathrm{nm} . \mathrm{km})$. At the receiver side, the signal is directly detected by an ideal highspeed PIN photodetector, amplified and each QAM channel is demodulated to produce 512 parallel data channels. These can be converted into a single data channel by parallel-to-serial conversion and down-converted to recover the $2.5 \mathrm{Gbit} / \mathrm{s}$ data. The optical field $E_{M Z}(t)$ at the output of the DD-MZM can be expressed as:

$$
E_{M Z}(t)=E_{i}(t)\left\{\eta\left[e^{j \frac{\pi V_{A 1}(t)}{V A_{\pi}}}+e^{j \frac{\pi V_{A 2}(t)}{V A_{\pi}}}\right]+\xi e^{j \frac{\pi V_{A 2}(t)}{V A_{\pi}}}\right\}
$$

Where $E_{i}$ is the optical field at the input of the DD-MZM and average power $P o, \mathrm{VA}_{\pi}$ is the switching voltage of the DD-MZM; $\eta$ and $\xi$ are given respectively by $\sqrt{r_{1} r_{2}}$ and 
$\sqrt{\left(1-r_{1}\right)\left(1-r_{2}\right)}-\sqrt{r_{1} r_{2}}$, where and $r_{1}$ and $r_{2}$ are the power splitting ratios of the input and output of the Y-junctions of the DD-MZM [7] respectively.

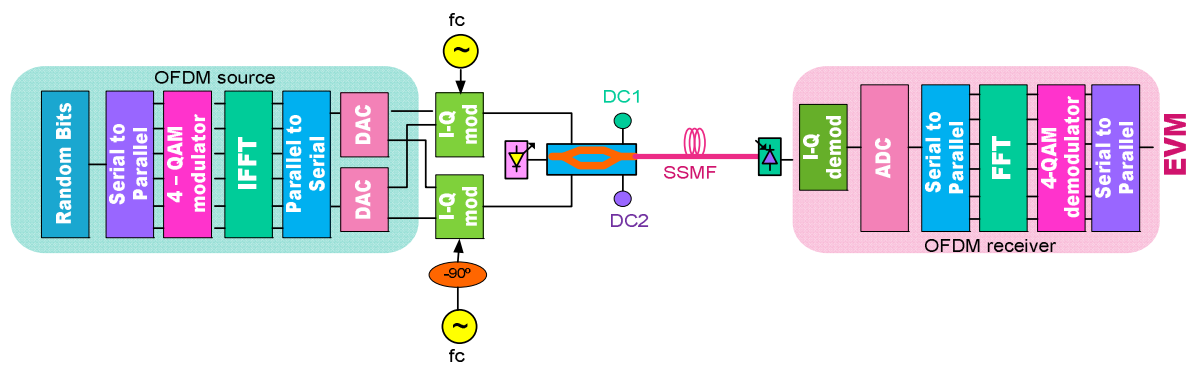

Fig. 1. System Architecture of a $60 \mathrm{GHz}$ OFDM-RoF with DD-MZM

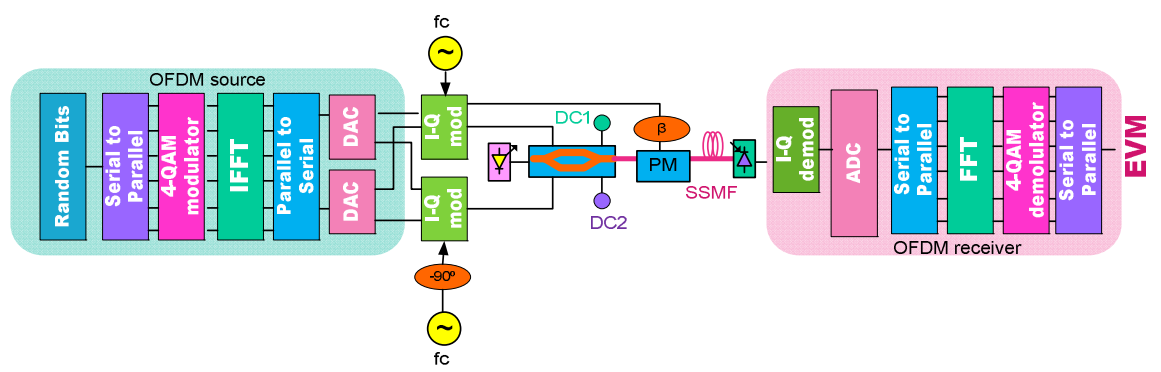

Fig. 2. System Architecture of a $60 \mathrm{GHz}$ OFDM-RoF with DD-MZM cascaded with a phase modulator

The electrical voltages applied on both electrodes of the DD-MZM modulator to obtain an OSSB signal consist of the sum of the OFDM signals, $s_{\text {OFDM }}(t)$ and a dc term, given by:

$$
\begin{aligned}
V_{A 1}(t) & =s_{\text {OFDM }}(t)-V A_{\pi} / 4 \\
V_{A 2}(t) & =\hat{s}_{\text {OFDM }}(t)+V A_{\pi} / 4
\end{aligned}
$$

Where $\hat{s}_{\text {OFDM }}(t)$ is the Hilbert transform of the OFDM signal.

In the case of DD-MZM cascaded with an optical phase modulator (PM) as illustrated in Fig. 3 the optical field $\mathrm{E}_{\mathrm{o}}$ is given by

$$
E_{o}(t)=E_{i}(t)\left\{\eta\left[e^{j \frac{\pi V_{A 1}(t)}{V A_{n}}}+e^{j \frac{\pi V_{A 2}(t)}{V A_{n}}}\right]+\xi e^{j \frac{\pi V_{A 2}(t)}{V A_{n}}}\right\} e^{j \frac{\pi V_{B}(t)}{V B_{n}}}
$$

While the voltage applied to the PM modulator is assumed to be $V_{B}(t)$; that is the modulated OFDM signal after being applied a adjustable phase shift $\beta+\pi / 2$. The objective of this phase shift is to eliminate the residual sideband due to DD-MZM finite extinction rate (ER). 


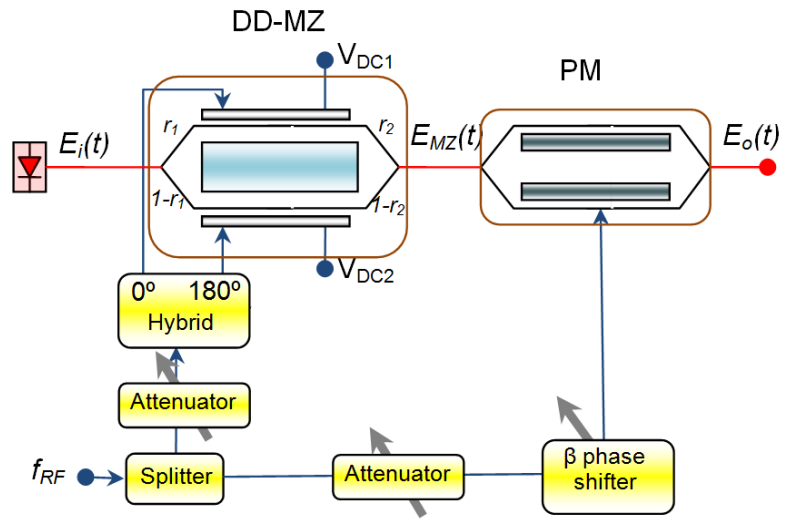

Fig. 3. Schematic of the ER compensation scheme

The principle of optical frequency up-conversion is to generate higher-order optical harmonics using an external nonlinear modulator, typically a Mach-Zehnder modulator (MZM) driven by a low frequency electrical signal, this reduces the bandwidth requirements for the optical modulator, the electrical drive signal source and the drive circuit itself. For example, for OSSB, frequency tripling is obtained by beating, upon square law detection by the photodetector, the $\omega_{c}$ harmonic with $\omega_{c}+3 \omega_{m}$, and all harmonics separated by $3 \omega_{m}$, where $\omega_{c}$ is the optical carrier frequency, and $\omega_{m}$ is the subcarrier frequency.

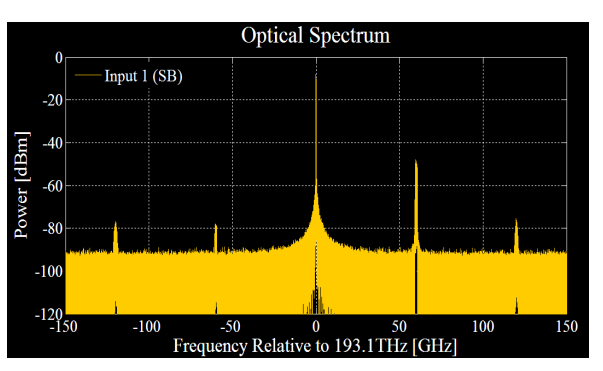

a)

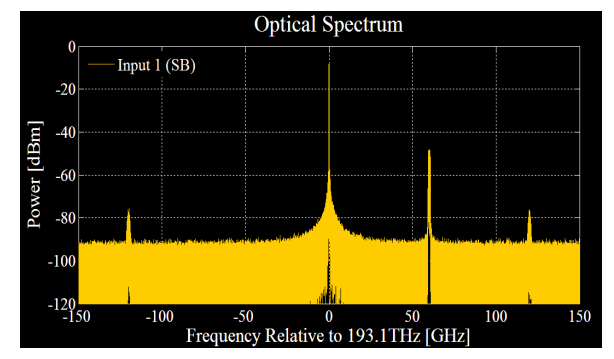

b)

Fig. 4. Optical power spectrum at a) Dual-Arm MZM (Rext=30dB)b) Dual-Arm with PM $(\mathrm{Rext}=30 \mathrm{~dB})$

For dual-arm MZM, the effect of the imbalance in the power splitting ratio generates odd low sideband harmonics as it is shown in Fig. 4a. For this situation, the imbalance leads to an optical spectrum that approaches an Optical Double Side Band (ODSB) signal. In the case of the DD-MZM cascaded with an optical phase modulator the optical spectrum approaches the ideal modulator spectrum (Fig 4b). 


\section{Simulation Results}

\subsection{Optical Modulation without Up-Conversion}

In order to compare the performance of both modulator schemes when digital data is transmitted, the DD-MZM and the DD-MZM cascaded with a phase modulator OSSB systems illustrated in Fig. 1 and 2 were simulated using the VPI TransmissionMak-er ${ }^{\mathrm{TM}}$ simulation platform. The simulation parameters were presented in section 3 . This includes the noise system, like RIN, shot noise and circuit noise. We named it the real circuit. We also performed an additional test to calculate the beta parameter in the phase modulator, in order to determine the angle at which the optical carrier in the lower sideband is completely suppressed. Intermodulation distortion that generates inter-subcarrier interference at fiber output arises mainly from the nonlinear characteristics of the optical modulator, the linear fiber dispersion and from the square law detection process.
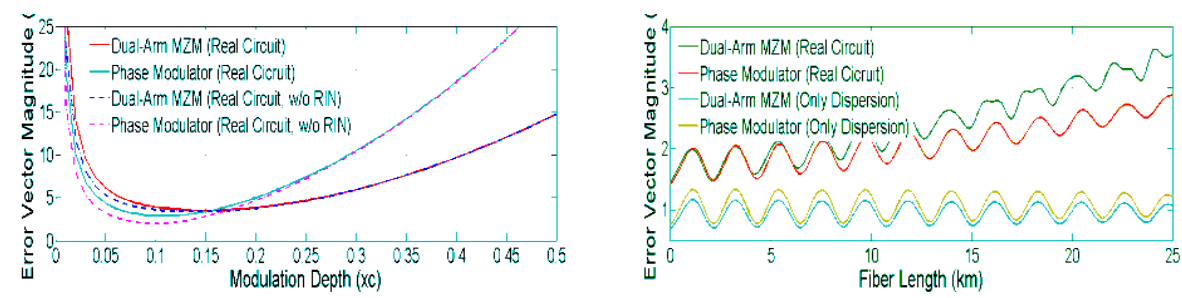

a)

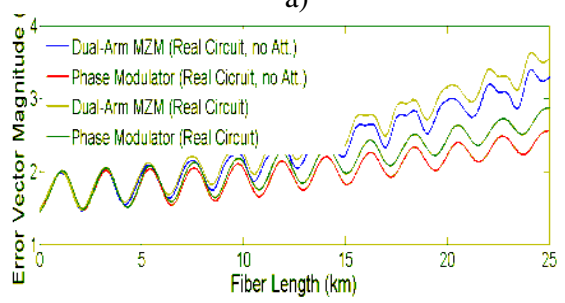

c)

d)

Fig. 5. EVM for DD-MZM and DD-MZM cascaded with PM versus a) modulation depth for $25 \mathrm{~km}$ of fiber length b) fiber length real conditions versus only dispersion c) fiber length real conditions versus without attenuation d) QAM constellation diagram

Figure 5.a illustrates the Error Vector Magnitude (EVM) versus modulation depth $\left(\mathrm{x}_{\mathrm{c}}\right)$ for $25 \mathrm{~km}$ of fiber length. We can conclude the optimal modulation depth is similar for all tested configurations. For lower modulation depths, the system noise is dominant, whereas for higher modulation depths the intermodulation distortion between subcarriers is dominant; this leads to an optimum modulation depth $\mathrm{x}_{\mathrm{c}}=0.155$, in the circuit with a DD-MZM, and $\mathrm{x}_{\mathrm{c}}=0.111$, in the circuit with a DD-MZM, plus a 
PM. From Fig 5.b, we can conclude the EVM does not change linearly with the fiber length. There are also periodic oscillations that result from the dispersion, making the EVM increase with the fiber length. This occurs due to the interplay between the laser RIN and the dispersion. When we don't have noise (only the dispersion) the EVM is periodic. Finally, in Fig.5.c, when we eliminate the attenuation we can see the EVM decreases slightly because the interplay between the RIN and the dispersion is dominant. This result can be confirmed in Fig. 5.d where the received constellation is presented (without attenuation) for different fiber lengths. From these results we concluded that OSSB based systems are robust against imperfect power splitting ratios and the use of an extra PM to eliminate the first low sideband harmonic is not necessary.

\subsection{Optical Modulation with Up-Conversion}

In this section we present the results obtained for the two modulation schemes studied, but using optical up-conversion with frequency tripling. The conditions are the same from last section and we use an electrical $20 \mathrm{GHz}$ to obtain $60 \mathrm{GHz}$ in frequency tripling. Comparing the performance of the systems using up-conversion with the ones from previous section, we can conclude that the results are significantly worse.

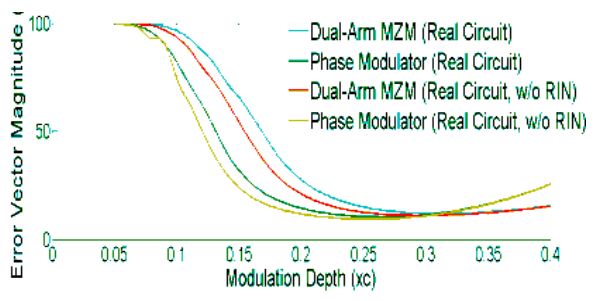

a)

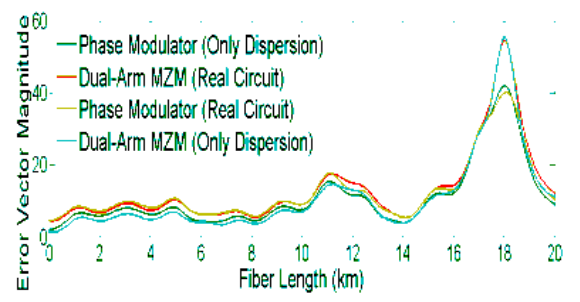

c)

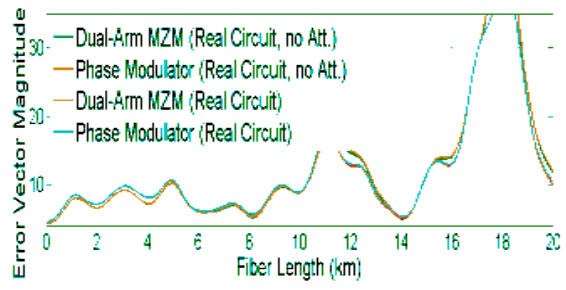

b)

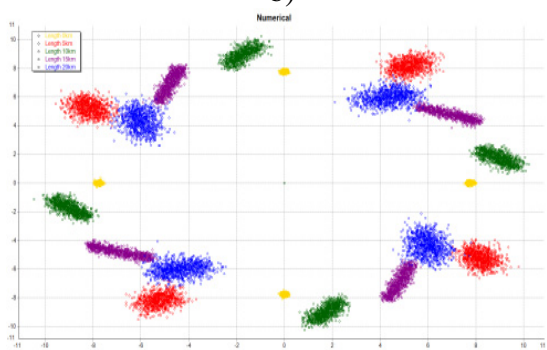

d)

Fig. 6. EVM for DD-MZM and DD-MZM cascaded with PM with up-conversion versus a) modulation depth for $25 \mathrm{~km}$ of fiber length b) fiber length real conditions versus only dispersion c) fiber length real conditions versus without attenuation d) QAM constellation diagram

This is explained on Fig. 6.b. and 6.c. In these figures we can see the dispersion is the main responsible for the performance degradation obtained. This occurs because up-conversion systems have different harmonics that suffer different dispersion 
effects when the fiber length increases.Furthermore, the beating during the photodetection process results in additional system degradation. This effect combined with the laser RIN can be seen in Fig. 6.d, where the receiver constellation is shown for different fiber lengths. We can conclude that this system needs equalization for distances beyond $15 \mathrm{~km}$. The use of the cascaded PM does not solve the problem.

\section{Conclusions and Further Work}

We concluded that OSSB based systems are robust against imperfect power splitting ratios and the use of an extra PM to eliminate the first low sideband harmonic is not necessary when $60 \mathrm{GHz}$ native frequency is used. When frequency tripling is used, this system needs equalization for distances beyond $15 \mathrm{~km}$. We also concluded that the dispersion is the mainly responsible for the performance degradation of the system when compared with the $60 \mathrm{GHz}$ natives. The use of the cascaded PM does not solve the problem.

In further work analytical methods will be developed to evaluate the degradation of performance of those systems caused by laser intensity noise at fiber output, intermodulation distortion and fiber dispersion. In case of optical up-conversion equalization techniques will be applied.

\section{References}

1. O’Reilly, J.J., Lane, P.M., Attard, J., Griffin, R.: Broadband wireless systems and networks: an enabling role for radio-over-fibre. Philosophical Transactions of the Royal Society a-Mathematical Physical and Engineering Sciences 358, 2297-2308 (2000)

2. Laurêncio, P., Medeiros, M.C.R.: Dynamic range of optical links employing optical singleside-band modulation. IEEE Photonics Technology Letters 15(5), 748-750 (2003)

3. Lin, C.T., Chen, J., Shih, P.T., Jiang, W.J., Chi, S.: Ultra-high data-rate $60 \mathrm{GHz}$ radio-overfiber systems employing optical frequency multiplication and OFDM formats. Journal of Lightwave Technology 28(16), 2296-2306 (2010)

4. Thakur, M.P., Medeiros, M.C.R., Laurêncio, P., Mitchell, J.E.: Optical frequency tripling with improved suppression and sideband selection. Optics Express 19, 457-468 (2011)

5. Hansen, C.J.: Wigig: Multi-Gigabit Wireless Communications in the $60 \mathrm{GHz}$ Band. In: IEEE Wireless Communications. Broadcom Corporation (December 2011)

6. IEEE802.15.3c WPAN Task Group 3c, http://www.ieee802.org/15/pub/ TG3 . html

7. Lin, C.T., Chen, J., Dai, S.P., Peng, P.C., Chi, S.: Impact of Nonlinear Transfer Function and Imperfect Splitting Ratio of MZM on Optical Up-Conversion Employing Double Sideband With Carrier Suppression Modulation. Journal of Lightwave Technology 26(15), 2449-2459 (2008) 